\title{
Philosophiques
}

\section{Le cognitivisme moral de Habermas fait-il face au problème de Frege-Geach?}

\section{Stéphane Courtois}

Volume 35, numéro 2, automne 2008

URI : https://id.erudit.org/iderudit/000443ar

DOI : https://doi.org/10.7202/000443ar

Aller au sommaire du numéro

Éditeur(s)

Société de philosophie du Québec

ISSN

0316-2923 (imprimé)

1492-1391 (numérique)

Découvrir la revue

Citer cet article

Courtois, S. (2008). Le cognitivisme moral de Habermas fait-il face au problème de Frege-Geach? Philosophiques, 35(2), 561-579.

https://doi.org/10.7202/000443ar
Résumé de l'article

L'article cherche à fournir une défense de la théorie discursive de la morale de Habermas contre une critique importante formulée récemment par J. G.

Finlayson, lequel soutient que Habermas rejetterait ce qu'il appelle le " cognitivisme métaéthique » et qu'un tel rejet le confronterait au problème de Frege-Geach. L'article démontre en détail que cette critique est non fondée. Il montre de plus que la seule forme de cognitivisme rejetée par Habermas est le descriptivisme moral en ce que cette approche serait contre-intuitive eu égard à l'usage normal de nos expressions morales. L'article cherche finalement à répondre à certaines objections majeures que les philosophes descriptivistes pourraient soulever à l'endroit de la théorie habermassienne de la morale, en particulier contre sa thèse de l'analogie entre vérité propositionnelle et justesse normative. 


\title{
Le cognitivisme moral de Habermas fait-il face au problème de Frege-Geach ?'
}

\author{
STÉPHANE COURTOIS \\ Département de philosophie \\ UQTR \\ stephane_courtois@uqtr.ca
}

\begin{abstract}
RÉSUMÉ. - L'article cherche à fournir une défense de la théorie discursive de la morale de Habermas contre une critique importante formulée récemment par J. G. Finlayson, lequel soutient que Habermas rejetterait ce qu'il appelle le « cognitivisme métaéthique » et qu'un tel rejet le confronterait au problème de Frege-Geach. L'article démontre en détail que cette critique est non fondée. II montre de plus que la seule forme de cognitivisme rejetée par Habermas est le descriptivisme moral en ce que cette approche serait contre-intuitive eu égard à l'usage normal de nos expressions morales. L'article cherche finalement à répondre à certaines objections majeures que les philosophes descriptivistes pourraient soulever à l'endroit de la théorie habermassienne de la morale, en particulier contre sa thèse de l'analogie entre vérité propositionnelle et justesse normative.
\end{abstract}

\begin{abstract}
The paper aims at providing a defence of Habermas's discourse theory of morality against a significant criticism recently levelled by J. G. Finlayson, who maintains that Habermas would reject what he calls "metaethical cognitivism" and that such a rejection would cause him to face what has been known as the Frege-Geach problem. The paper demonstrates in detail that this claim is unfounded. It further shows that the only form of cognitivism rejected by Habermas is moral descriptivism, since this approach would be counter-intuitive as regards the normal use of our moral expressions. The paper finally seeks to respond to major objections descriptivist philosophers might raise against Habermas's theory of morality, in particular against his analogy thesis between propositional truth and normative rightness.
\end{abstract}

Dans les pages qui suivent, j'aimerais offrir une réplique à un article de J. G. Finlayson paru récemment ${ }^{2}$ se présentant comme une critique de l'éthique de la discussion et de la théorie pragmatique de la vérité de Habermas. Finlayson défend deux thèses principales. Premièrement, il soutient que la théorie discursive de la morale de Habermas rejetterait ce qu'il appelle le

1. Nicolas Kaufman était un philosophe capable de se mouvoir avec une grande facilité à l'intérieur des traditions philosophiques européenne/continentale et analytique/angloaméricaine. Ce texte porte sur un auteur, Jürgen Habermas, dont il connaissait très bien les travaux, et tente de faire converger ces deux traditions qui lui étaient chères.

2. J. G. Finlayson. (2005) «Habermas's Moral Cognitivism and the Frege-Geach Challenge ", European Journal of Philosophy, 13, p. 319-344. 
«cognitivisme métaéthique» — selon lequel les énoncés moraux sont susceptibles de vérité et expriment des croyances qui aspirent à la vérité - et qu'elle n'endosserait qu'un cognitivisme moral plus faible où les expressions morales soulèvent une prétention à la "justesse ", non à la vérité, la première n'étant qu'un analogue de la seconde. La seconde thèse de Finlayson est que, étant donné le rejet par Habermas du cognitivisme métaéthique, sa théorie discursive de la morale se confronterait à ce que Finlayson appelle le problème de Frege-Geach (Frege 1970; Geach 1965), stipulant qu'il est impossible d'expliquer la validité d'un raisonnement quel qu'il soit sans faire appel à la notion de vérité, ce qui placerait les défenseurs du non-cognitivisme métaéthique dans une position indéfendable.

Dans cet article, j'examine chacune des deux thèses de Finlayson et démontre qu'elles sont non fondées. Je commence par la seconde thèse, laissant temporairement de côté la question de savoir si la théorie discursive de la morale de Habermas souscrit ou non au cognitivisme métaéthique de Finlayson. Je démontre que le problème de Frege-Geach n'affecte aucunement la conception habermassienne de la morale et ce, quelles que soient les raisons que Finlayson a à l'appui de sa position, que ce soit parce que, selon lui, la théorie discursive de la morale substituerait erronément la notion de justesse normative au critère traditionnel de vérité ou parce qu'elle s'appuierait sur une lecture pragmatique douteuse de la notion de vérité en lieu et place d'une interprétation sémantique plus traditionnelle. Dans la seconde partie de mon texte, je reviens à la première thèse de Finlayson, prétendant que la théorie discursive de la morale de Habermas souscrit à une sorte de non-cognitivisme métaéthique. Je maintiens plutôt que ce qui est rejeté par l'éthique de la discussion n'est pas le cognitivisme moral, quel que soit le sens qu'on lui donne, mais le descriptivisme moral, énonçant que le rôle des jugements moraux est de décrire des faits de la même manière que les énoncés descriptifs et, pour cette raison, que les premiers ont à être évalués de la même manière que les seconds. Je démontre que, si la thèse de Finlayson est que le cognitivisme moral n'est possible que sous la forme du descriptivisme moral, en d'autres mots, si la prétention à la vérité des jugements moraux ne peut être comprise autrement que comme la prétention à la vérité des énoncés descriptifs, alors cela risque de distordre l'usage normal de nos expressions morales et le sens que nous leur accordons, provoquant d'importantes difficultés que la théorie discursive de la morale de Habermas a précisément pour objectif de résoudre. Dans la dernière partie de l'article, j'examine les vrais défis que, à mon sens, ne peut éviter de rencontrer la thèse habermassienne de l'analogie entre "vérité propositionnelle » et « justesse normative ». J'analyse deux objections éventuelles de la part des descriptivistes contre une telle distinction et je réplique à chacune d'elles. 
Pour savoir si le problème de Frege-Geach (FG) s'applique ou non à la conception habermassienne de la morale, il convient au préalable de rappeler l'objectif poursuivi par FG, lequel FG a pour objectif initial d'ébranler les assises du subjectivisme moral et de l'anti-cognitivisme, positions qui s'expriment dans des doctrines comme l'émotivisme (Ayer 1936), l'expressivisme (Stevenson 1944), ou le projectivisme moral (Blackburn 1984). Selon toutes ces doctrines, les jugements moraux que nous exprimons habituellement dans des énoncés évaluatifs n'auraient aucun contenu cognitif réel. Ils auraient une forme descriptive de surface, mais, au fond, ils ne seraient rien d'autre qu'un véhicule parmi d'autres destiné à extérioriser nos sentiments d'approbation ou de désapprobation. Par exemple, la proposition «Torturer les animaux est mal » aurait, dans le langage émotiviste ou expressiviste, une signification purement émotive et devrait être retraduite par quelque chose comme «Honte à la personne qui torture les animaux ! ». Le problème cependant est que la première proposition peut être dite vraie ou fausse, non la seconde. Et des difficultés apparaissent lorsque des expressions du second type sont enchâssées dans des raisonnements contenant des négations et des énoncés de forme conditionnelle. Alors que les énoncés évaluatifs du premier type fonctionnent normalement dans nos raisonnements à l'instar de n'importe quel autre énoncé descriptif ordinaire, les inférences logiques deviennent impossibles avec les expressions émotives du second type, et cela apparaît plutôt contre-intuitif compte tenu de notre usage ordinaire du raisonnement moral. Finalement, FG somme les subjectivistes moraux et les émotivistes de tout acabit de fournir une réponse cohérente à de telles anomalies et les oblige à clarifier leurs vues.

Il est loin d'être sûr que les difficultés mises à jour par FG s'appliquent réellement à la conception habermassienne de la morale. Rappelons que ce que Habermas propose est une conception déontologique de la morale, où l'analyse des jugements moraux se concentre, non sur les énoncés évaluatifs (de la forme " $X$ est courageux, vil, sage, ignoble », etc.), mais sur les énoncés normatifs (de la forme "Il est obligatoire, interdit, permis que $p$ ", ou "de faire $X »)$. À la différence des émotivistes, des expressivistes ou des projectivistes, Habermas ne croit aucunement que le langage de la morale ne soit qu'une simple expression de nos attitudes subjectives et de nos émotions. La thèse standard de l'éthique de la discussion est plutôt que l'on doit opérer une distinction entre les énoncés évaluatifs et les énoncés normatifs, entre le bien et le juste, entre les questions pratiques ayant à voir avec la "vie bonne ", ou avec les valeurs et les préférences individuelles, et celles appartenant à la justice. La thèse centrale de l'éthique de la discussion est que seules les normes (qu'il s'agisse de requêtes, d'ordres, de commandements, de prohibitions, de permissions, etc.) sont en mesure d'exprimer des intérêts généralisables et de mériter l'accord de tous ceux participant à un discours pratique, mais non les énoncés évaluatifs (Habermas 1990 : 108; 1993 : 91; 1996 : 97, 161-2). 
En somme, la conception déontologique de la morale mise en avant par l'éthique de la discussion ne cherche aucunement à remplacer les expressions évaluatives par d'autres expressions purement émotives et non susceptibles de vérité, tout en prétendant que ces dernières formeraient la structure profonde des premières. Sa thèse est plutôt que les énoncés normatifs forment, à côté des énoncés évaluatifs, une composante centrale du langage de la morale qui doit être prise au sérieux dans la mesure où il s'agirait de la sorte d'expressions morales les plus susceptibles d'un consensus rationnellement motivé.

Bref, pour rester fidèle à la conception déontologique de la morale de Habermas, un raisonnement moral qui aurait à passer le test que présente FG devrait en principe enchâsser des énoncés normatifs. Prenons l'exemple suivant :

1. Il est obligatoire de rembourser ses emprunts, alors rembourser ses emprunts est permis.

2. Il est obligatoire de rembourser ses emprunts.

3. Rembourser ses emprunts est permis.

Le raisonnement précédent n'éprouve aucun problème particulier avec FG et il rencontre le défi qu'il lance parfaitement. Premièrement, le raisonnement est formellement correct selon les standards usuels de la logique déontique, ce qui ne serait pas le cas si une prémisse, disons la prémisse (2), était remplacée par une expression émotive comme "Honte à tous ceux qui ne remboursent pas leurs emprunts!». Mais nous ne rencontrons pas une telle difficulté ici. Ensuite, la proposition de la prémisse (2) a la même signification que celle qui sert à former l'énoncé conditionnel de la prémisse (1). C'aurait été différent si la prémisse (2) avait eu un sens émotif. Mais nous ne rencontrons pas une telle difficulté non plus. Finalement, nous n'avons aucune raison de rejeter la conclusion (3) après avoir accepté les prémisses (1) et (2), ce qui aurait été différent si l'une de ces prémisses contenait une expression ayant un sens émotif. Mais ce n'est pas le cas ici. Alors, où est le problème?

Finlayson a deux raisons principales qui l'autorisent à penser que l'éthique de la discussion ne passe pas le test que présente FG. J'examine chacune d'elles séparément.

(1) La première raison est que, selon l'éthique de la discussion, les énoncés normatifs soulèvent une prétention à la « justesse » (Richtigkeit), non à la «vérité ». Cependant, selon Finlayson (2005: 326-7), la validité d'un raisonnement moral syntaxiquement structuré sous la forme d'un modus ponens $\left([(p) q){ }_{-} p\right](q)$, comme dans notre exemple, ne peut être expliqué si l'on ne fait pas appel à la notion de vérité. À partir du moment où l'on a recours à une notion différente de la validité pour tenir compte du discours moral, la question se pose de savoir de quelle manière la validité d'un raisonnement moral quel qu'il soit peut être expliquée. Pourquoi, se demande Finlayson, le type de raisonnement moral exposé plus haut serait-il préservateur de vérité quant à sa structure syntaxique, logico-formelle, mais ne serait 
plus préservateur de vérité lorsque mesuré à l'aune de la norme de justesse? Ainsi, de la même manière que les subjectivistes doivent expliquer comment des énoncés évaluatifs peuvent fonctionner correctement dans un raisonnement moral s'ils ne sont autre chose que l'expression de nos attitudes d'approbation ou de désapprobation, Habermas se devrait d'expliquer comment des énoncés normatifs (ou toute autre expression morale) peuvent être enchâssés dans un raisonnement formellement valide si une telle validité formelle ne peut être expliquée en faisant appel à la notion de vérité, mais seulement à une exigence de justesse.

Il ne fait aucun doute que la thèse de l'analogie entre vérité et justesse, qui est centrale pour l'éthique de la discussion, pose des difficultés importantes, et j'entends revenir sur ce point plus tard. Cependant, il est loin d'être clair qu'une telle thèse expose l'éthique de la discussion aux types de difficultés auxquelles se butent les subjectivistes moraux lorsque confrontés à FG. Pour échouer le test que présente FG, deux conditions doivent être rencontrées: (1) il faut rejeter ce que Finlayson appelle le "cognitivisme métaéthique ", c'està-dire la conception selon laquelle les énoncés moraux sont susceptibles de vérité et expriment des croyances qui aspirent à la vérité ; 2 un tel rejet doit entraîner l'incapacité (réelle ou apparente) des expressions morales de fonctionner convenablement dans un raisonnement valide. Les théories morales subjectivistes, comme l'émotivisme, l'expressivisme ou le projectivisme, sont directement mises au défi par FG parce qu'elles rencontrent les deux conditions. Mais il est loin d'être sûr que l'éthique de la discussion fait aussi mal. Même en concédant pour les fins de l'argument qu'elle rencontre la condition (1) (ce qui reste à prouver, comme je le montrerai plus loin), cela ne signifie pas automatiquement qu'elle rencontre la condition (2). Comme je l'ai montré plus haut, les énoncés normatifs s'enchâssent parfaitement bien dans tout raisonnement moral valide et, contrairement aux expressions émotives, ils ne nuisent pas à notre capacité à faire des négations ou à formuler des énoncés conditionnels.

Bien entendu, ce que Finlayson juge discutable dans les positions que défend l'éthique de la discussion, ce n'est pas la façon dont la délibération morale doit opérer une fois que les énoncés descriptifs sont remplacés par des énoncés normatifs, comme c'est le cas avec les expressions émotives, mais plutôt la façon dont les propriétés formelles du discours moral ont à être évaluées à partir du moment où l'on passe d'un critère de vérité à la norme de justesse. Cependant, Finlayson me semble commettre l'erreur de soupçonner l'éthique de la discussion de vouloir mesurer le raisonnement moral en entier à la lumière d'une norme de justesse, alors qu'elle n'entretient aucunement cette prétention. Le critère de justesse normative n'a pas pour but de mesurer les traits syntaxiques du discours moral en général, mais uniquement les prétentions spécifiques que font valoir les jugements normatifs par rapport aux jugements descriptifs. Ainsi, l'affirmation selon laquelle les expressions normatives doivent être évaluées à l'aune d'une norme de justesse quant à leur contenu 
est parfaitement compatible avec l'idée que les traits formels ou syntaxiques du raisonnement moral, ou de quelque raisonnement que ce soit, soient de leur côté évaluables à la lumière d'un critère de vérité indépendant. En fait, la même chose s'applique aux énoncés descriptifs eux-mêmes. Alors que toutes les propositions d'un raisonnement peuvent se révéler parfaitement fausses quant à leur contenu empirique, le raisonnement peut néanmoins s'avérer valide (ou analytiquement vrai) quant à ses propriétés formelles. Finlayson soutiendrait-il qu'un tel raisonnement échoue le test proposé par FG ? Sûrement pas. Alors, pourquoi la même chose ne vaudrait-elle pas pour le raisonnement moral, comme dans notre exemple? Il est possible que les propositions normatives qu'il contient expriment des croyances ou prescrivent des actions jugées moralement condamnables à l'aune d'un certain critère de validité (quelle que soit son appellation: "justesse normative", "vérité morale ", "rectitude morale ", etc.), tout en étant enchâssées dans un raisonnement que l'on estime formellement correct à la lumière d'un autre critère de validité (par exemple, un critère sémantique-formel de vérité). Ce que ces réflexions montrent est que le défi lancé par FG a moins à voir avec le critère de validité utilisé pour évaluer le contenu des énoncés, qu'ils soient empiriques ou normatifs, qu'avec leur capacité à s'emboîter formellement dans des relations logiques sans que cela n'affecte l'usage normal du raisonnement. Si les théories morales subjectivistes échouent à relever ce défi, il ne pose à l'éthique de la discussion aucune difficulté particulière.

(2) Mais Finlayson ne se contente pas seulement de reprocher à l'éthique de la discussion d'être incapable d'expliquer la validité formelle du raisonnement moral lorsqu'elle en appelle à la norme de justesse. Il (2005 : 329-30) lui reproche également d'être intriquée dans un cercle vicieux dans la mesure où elle n'aurait d'autre choix que de devoir recourir à une notion sémantique de vérité qui est plus primitive que la lecture pragmatique qu'elle suggère de cette même notion. Si je comprends Finlayson correctement, l'éthique de la discussion ferait face dans ce cas aux difficultés posées par FG, non plus parce qu'elle substitue une notion de justesse normative au critère traditionnel de vérité, rendant ainsi les traits formels du raisonnement moral difficiles à évaluer correctement, mais parce qu'elle cherche à développer une lecture pragmatique de la notion de vérité en lieu et place d'une lecture sémantique plus traditionnelle, tout en n'ayant d'autre choix que de devoir présupposer cette dernière, ce qui la mettrait en contradiction avec elle-même. J'ai expliqué pourquoi la première raison de croire que l'éthique de la discussion succombe aux difficultés de FG n'est pas fondée. Je me tourne maintenant vers la seconde.

L'éthique de la discussion intègre les composantes syntaxiques du raisonnement discutées plus haut à l'intérieur des règles de la discussion, elles appartiennent donc à ce que Habermas (1990: 86-94) nomme les présuppositions pragmatiques de l'argumentation. Cependant, au nombre des règles de la discussion figurent, non seulement ces règles syntaxiques et sémantiques de base destinées à assurer la cohérence des discours, mais aussi des règles de 
sincérité (telles que: "Tout locuteur ne peut affirmer que ce qu'il croit réellement»), et d'autres règles, les plus importantes pour l'éthique de la discussion, ayant une portée normative (telles que : "Tous les locuteurs compétents sont autorisés à prendre part aux discours »). Pour quelle raison l'éthique de la discussion serait-elle piégée dans un dilemme? Ce serait, selon Finlayson, parce qu'elle met explicitement en avant un concept pragmatique de vérité alors qu'elle se voit obligée, pour spécifier les règles pragmatiques du discours, de s'appuyer implicitement sur un concept sémantique de vérité, tout en étant incapable de préciser lequel des deux est le plus primitif dans la théorie. Ou bien la notion générale de "validité " (Gültigkeit, Geltung) développée par la théorie de l'activité communicationnelle est primitive et la vérité (Wahrheit) n'en est qu'une spécification particulière, à côté de la justesse (Richtigkeit) et de la véracité (Wahrhaftigkeit) et, comme ces dernières, la vérité forme une notion essentiellement pragmatique, c'est-à-dire une prétention à la validité (Geltungsanspruch) qui doit être honorée à l'intérieur d'un discours. Mais alors, selon Finlayson, Habermas se voit dans l'obligation de fournir une théorie discursive ou pragmatique de la notion sémantique de vérité qu'il utilise pour spécifier les règles du discours elles-mêmes, ce qui apparaît vicieusement circulaire. Ou bien la vérité est plus qu'une notion pragmatique, plus qu'une simple spécification, à côté de la justesse et de la véracité, de la notion générale de validité, puisque l'éthique de la discussion doit recourir à une notion plus primitive de vérité de nature sémantique et la présupposer, auquel cas l'entreprise de Habermas est ébranlée.

Â mon sens, ce type de dilemme n'est qu'une autre manière de formuler le fameux débat entre Habermas et Karl-Otto Apel, lequel est devenu un lieu commun pour tous ceux qui sont le moindrement familiers avec les travaux de la seconde génération de l'école de Francfort, débat dont l'enjeu central est le statut des énoncés philosophiques fondamentaux de la pragmatique universelle ou de l'éthique de la discussion. Comme ces disciplines ont pour mission d'exposer les conditions idéales de délibération dans lesquelles la vérité des propositions ou la justesse des normes sont examinées, leurs propres énoncés ne peuvent pas eux-mêmes se soumettre circulairement aux conditions de justification idéale qu'ils cherchent à expliciter. Quel est alors leur statut ? Pour Habermas (1990 : 94-98; 1998 : 41-46), ils ont un statut « quasitranscendantal». De manière comparable aux jugements synthétiques a priori de Kant, ils sont nécessaires et ont une portée universelle. Habermas estime cependant qu'ils demeurent "factuellement" incontestables en ce qu'il n'existe présentement pour eux aucune solution viable de remplacement, sans que l'on puisse toutefois exclure la possibilité qu'un jour nous changions notre façon de concevoir le monde. En conséquence, bien qu'en principe nécessaires et universels, les énoncés philosophiques fondamentaux de la pragmatique universelle ou de l'éthique de la discussion sont aussi faillibles que les énoncés empiriques. Ils requièrent eux aussi une justification dans un discours et 
ont, par le fait même, à rencontrer les mêmes conditions pragmatiques de l'argumentation que n'importe quelle autre proposition. Ils ne sont fondés sur aucun métadiscours, aucune métathéorie. Il n'existe, selon Habermas (1986: 350), aucune hiérarchie dans les niveaux de discours, mais qu'un seul lieu pour la justification de toutes nos expressions quelles qu'elles soient, celui des discours argumentés. Cette position radicalement anti-fondationnaliste contraste nettement avec celle adoptée par Apel $(1987$; 1989; 1998a ; 1998b ; 1998c), qui reproche depuis des années à Habermas, dans un esprit analogue à Finlayson, de persister dans l'auto-contradiction. Apel juge qu'une position cohérente doit distinguer deux classes d'énoncés: les énoncés empiriques des sciences (sociales et naturelles), qui sont fondamentalement révisables et requièrent pour cette raison une justification rationnelle dans un discours; et les énoncés pragmatiques-transcendantaux de la philosophie, qu'il juge universels, a priori valides et non révisables, leur rôle étant de mettre à jour les règles des discours et les présuppositions pragmatiques de l'argumentation rationnelle. De ce point de vue, la théorie pragmatique de la vérité serait appropriée pour la première classe d'énoncés, non pour la seconde. En effet, la " vérité » des énoncés pragmatiques-transcendantaux ne peut selon Apel être contestée par un locuteur sans qu'il ne fasse récursivement appel à elle, et donc sans qu'il ne commette de contradiction performative, et elle ne peut non plus être fondée sur un principe supérieur sans que ce principe ne présuppose luimême la vérité de ces énoncés, et donc sans pétition de principe. En conséquence, les énoncés pragmatiques-transcendantaux de la philosophie n'auraient pas à se soumettre eux-mêmes aux normes du discours. Leur "vérité " aurait à se mesurer, non plus à la force du meilleur argument, mais à la certitude réflexive acquise au moyen d'une réflexion transcendantale sur les conditions ultimes de la rationalité et de l'argumentation rationnelle.

Qui a raison dans cette controverse ? Ce n'est certainement pas mon intention de trancher la question dans cet article. Mais quelle que soit la réponse que l'on donne aux questions difficiles en jeu ici, il est loin d'être clair que l'éthique de la discussion soit ébranlée par les difficultés posées par FG, tout au moins dans le sens que leur accorde cette fois Finlayson, celui d'une autocontradiction provenant de l'impossibilité d'éviter la notion sémantique de vérité. Supposons un moment que la théorie pragmatique de la vérité de Habermas ne soit pas autosuffisante, qu'il n'ait d'autre choix que d'abandonner sa prétention à l'universalité et qu'il doive, lorsqu'il cherche à rendre compte des règles du discours, en appeler à une autre notion de vérité (sémantique selon Finlayson, transcendantale selon Apel) plus primitive ou fondamentale. Est-ce que la reconnaissance de cette difficulté devrait obligatoirement forcer Habermas à renoncer à sa théorie ? Rien ne l'y oblige. Habermas pourrait très bien continuer de considérer sa théorie pragmatique de la vérité comme correcte, mais choisir de limiter sa portée, de la cantonner à ce que nous pourrions appeler des énoncés (empiriques ou normatifs) de premier niveau. Il pourrait très bien développer pour les énoncés philosophiques centraux de 
la pragmatique universelle et de l'éthique de la discussion une théorie de la vérité du second ordre (la théorie réflexive-transcendantale d'Apel s'offre à titre d'exemple, mais il n'est pas impossible de penser que d'autres conceptions de la vérité d'inspiration davantage sémantique puissent également se porter candidates). On sait très bien que cette voie est impraticable pour Habermas étant donné ses positions officiellement anti-fondationnalistes. Mais supposons un moment qu'il n'ait d'autre choix que d'emprunter cette voie pour éviter l'auto-contradiction. Une théorie pragmatique de la vérité (pour les énoncés empiriques et normatifs ordinaires) et une autre, d'inspiration davantage sémantique ou transcendantale (pour les énoncés philosophiques fondamentaux) pourraient très bien cohabiter sans distorsion (il s'agit en fait de la stratégie adoptée par Apel). Si l'idée maîtresse derrière FG est que tous les énoncés doivent ultimement se conformer à une certaine notion sémantique de vérité, cette thèse n'est pas exclusive. Elle peut se combiner à d'autres thèses qui conviennent davantage à la notion pragmatique de vérité. En tout cas, il n'est certainement pas nécessaire de recommander à Habermas, comme le fait Finlayson (2005: 330-6), d'abandonner sa théorie pragmatique en totalité en faveur d'une théorie déflationniste de la vérité ou d'une attitude agnostique envers la vérité. Si Habermas se montre réticent à l'endroit des conceptions de la vérité plus robustes (qu'elles soient de nature sémantique ou transcendantale), la solution minimaliste que lui suggère Finlayson est sûrement encore moins attrayante pour lui.

\section{II}

J'ai cherché jusqu'ici à montrer que les difficultés soulevées par FG, quel que soit le sens que leur donne Finlayson ou les raisons qu'il a de penser que l'entreprise de Habermas se confronte à elles, n'affectent, ni la thèse de l'analogie entre vérité et justesse mise en avant par l'éthique de la discussion ni la conception pragmatique de la vérité sur laquelle elle s'appuie. J'entends maintenant examiner la première thèse avancée par Finlayson, la plus importante à mon sens, selon laquelle l'éthique de la discussion rejetterait ce qu'il appelle le cognitivisme métaéthique. Si cette thèse m'apparaît la plus importante, c'est que la valeur de la seconde thèse de Finlayson que nous avons examinée, prétendant que l'éthique de la discussion serait victime des difficultés posées par FG, dépend en quelque sorte de la plausibilité de la première thèse. Pour pouvoir prétendre que l'éthique de la discussion se confronte à FG, il faut en effet admettre comme fondée la thèse selon laquelle elle conteste que les énoncés moraux soient susceptibles de vérité ou expriment des croyances qui aspirent à la vérité. Mais est-ce le cas ? J'ai laissé la question en suspens jusqu'ici. J'entends maintenant examiner cette thèse plus en détail, indépendamment de ses relations aux difficultés posées par FG.

L'idée centrale de l'éthique de la discussion n'est aucunement que les jugements moraux et les énoncés normatifs ne sont pas susceptibles de vérité, mais qu'ils ne sont pas susceptibles de vérité dans le même sens que les énoncés 
descriptifs. Ce que l'éthique de la discussion rejette n'est pas le cognitivisme moral en quelque sens que ce soit, mais le descriptivisme moral, c'est-à-dire la conception selon laquelle le sens des expressions morales est à chercher dans leur fonction descriptive, consistant à attribuer certaines propriétés (bon, juste, obligatoire, etc.) à certaines catégories de sujets (agents, actions, institutions, etc.). La thèse centrale du descriptivisme moral est que les jugements moraux ne sont qu'une sorte parmi d'autres de jugements déclaratifs, qu'ils fonctionnent syntaxiquement et sémantiquement de la même manière et, en conséquence, qu'ils ont à être évalués de manière identique, selon un même critère de vérité.

L'originalité de l'éthique de la discussion est que, de la même manière que la plupart des doctrines descriptivistes (comme le naturalisme moral ou l'intuitionnisme moral) et contrairement à la plupart des doctrines subjectivistes (comme l'émotivisme et l'expressivisme), elle défend une forme de cognitivisme moral. Cependant, contrairement au descriptivisme, elle soutient que la fonction des jugements moraux n'est pas de décrire des faits dans le monde externe mais de réguler normativement les actions humaines. En conséquence, les jugements moraux ne sont pas une sorte particulière de jugements déclaratifs, ils appartiennent plutôt à la classe des expressions déontiques, prescriptives ou normatives et ont à être évalués en tant que tels. En un mot, l'éthique de la discussion propose une théorie morale cognitiviste, mais non descriptiviste.

La question se pose maintenant de savoir si le fait de refuser le descriptivisme moral entraîne le rejet de ce que Finlayson appelle le "cognitivisme métaéthique ». Cela dépend. Si par "cognitivisme métaéthique », Finlayson entend le "descriptivisme moral ", il est clair que Habermas rejette cette position. Ce qui est moins clair, c'est de savoir si le " cognitivisme métaéthique », c'est-à-dire la conception selon laquelle les jugements moraux sont susceptibles de vérité et expriment des croyances qui aspirent à la vérité, implique nécessairement le descriptivisme, à plus forte raison si l'on défend, comme Habermas, une conception déontologique de la morale où l'analyse des jugements moraux se centre, non sur les énoncés évaluatifs, mais sur les énoncés normatifs. Si les énoncés évaluatifs ont un sens descriptif et possèdent la même structure logique que les énoncés descriptifs ordinaires, tel n'est pas le cas pour les énoncés normatifs. L'énoncé « On ne doit pas attaquer les innocents " possède un contenu propositionnel comme tous les énoncés descriptifs sans cependant avoir un sens descriptif, ni même une structure prédicative. Malgré ces différences, il fait toujours sens de demander si une telle proposition est vraie ou fausse. La question qui demeure ouverte est: en quel sens?

Les philosophes moraux descriptivistes ont jusqu'à maintenant adopté deux stratégies pour contourner les complications liées aux jugements normatifs. J'appellerai la première stratégie la stratégie "éliminativiste », qui est à mon sens la moins prometteuse. Elle consiste à bannir les jugements normatifs de l'éventail de nos expressions morales authentiques pour la raison 
que, comme le prétendent les partisans du particularisme éthique comme Jonathan Dancy (2004), les principes moraux d'ordre général n'auraient pas de valeur morale véritable et leur prétention à l'universalité serait illusoire. J'appellerai l'autre stratégie, plus familière, la stratégie réductionniste. Elle consiste simplement à convertir les expressions déontiques sous la forme d'énoncés descriptifs de manière à faciliter leur analyse en termes de vérité ou de fausseté. Par exemple, l'expression «Il est obligatoire que $p$ » — où "obligatoire » fonctionne comme un opérateur déontique appliqué à une proposition - peut être converti sous la forme " $x$ est obligatoire » ou «faire $x$ est obligatoire » — où "obligatoire» fonctionne désormais comme un prédicat ordinaire. Je soupçonne Finlayson d'avoir un penchant pour cette stratégie pour autant qu'il semble souhaiter que les expressions morales s'ajustent à la forme descriptive standard. Le but principal de son article — soumettre l'éthique de la discussion au défi que représente FG — milite en faveur de cette hypothèse. Mais alors, cela signifie qu'avec son " cognitivisme métaéthique », ce que Finlayson a à l'esprit est le "descriptivisme moral ", doctrine officiellement rejetée par Habermas. Et si la thèse véritable de Finlayson est que le cognitivisme moral n'est possible que sous la forme du descriptivisme moral, que les prétentions à la vérité des jugements moraux ne peuvent être comprises autrement que comme les prétentions à la vérité d'énoncés descriptifs, on doit alors se demander si une telle position ne risque pas de provoquer certaines distorsions dans l'usage normal de nos expressions morales et le sens que nous leur donnons. La critique proposée par Finlayson de l'éthique de la discussion n'aurait pour résultat final que de remplacer les difficultés qu'elle entendait initialement mettre à jour et surmonter par d'autres difficultés, peut-être plus substantielles. Dans ce qui suit, je mentionnerai seulement deux difficultés posées par la stratégie réductionniste.

La première difficulté est, il me semble, de méconnaître la part d'irréductibilité des énoncés normatifs par rapport aux énoncés descriptifs, en d'autres termes, de méconnaître le fait que toute tentative visant à reconfigurer les énoncés normatifs sous une forme descriptive risque de conduire à des distorsions dans la compréhension que nous avons de nos expressions morales et du sens proprement déontologique des normes : " $X$ est obligatoire » (dans telle institution, telle société, etc.) n'a pas la même signification que "Il est obligatoire de faire $x »$. Dans un cas, on se demande si la proposition est vraie, s'il s'agit là d'un fait, dans l'autre, si la norme est juste, s'il est moralement correct de recommander cette règle d'action de manière générale. Bref, convertir les énoncés normatifs en énoncés descriptifs et considérer la vérité propositionnelle comme le critère unique d'évaluation des énoncés tant descriptifs que normatifs risque de restreindre le cadre d'analyse des expressions morales à celui des relations énoncés / monde, où le « monde » est entendu ici au sens de l'ensemble des états de choses existants, avec cette conséquence que les normes risquent de se voir ramener au statut de simples états de choses (par exemple à des faits sociaux). Les travaux de Habermas relatifs à la 
pragmatique universelle permettent d'éviter une telle interprétation réductrice en élargissant les présuppositions ontologiques de la théorie sémantique du langage. Ils développent l'idée d'un système de «mondes » communs aux locuteurs et aux auditeurs - que nous pourrions appeler des référents "pragmatiques » - qui ont pour fonction de connecter les autres modes d'utilisation du langage que celui consistant à décrire des faits - tel que réguler normativement les relations interpersonnelles - à certains systèmes de référence. Ils proposent ainsi de différencier le monde externe des états de chose existants en monde "objectif » - accessible par la perception et l'observation et en monde "social» — se présentant de son côté comme une réalité normative accessible par l'attitude performative des locuteurs et dont les composantes morales deviennent perceptibles par la réflexion et la délibération ${ }^{3}$.

La seconde difficulté que je vois à la stratégie réductionniste adoptée par certains descriptivistes est de tenter, sans doute avec raison, d'assurer une certaine continuité entre le langage descriptif et le langage moral, d'éviter un dualisme trop tranché et infructueux entre raison théorique et raison pratique, mais de le faire au mépris de la différence subsistant entre les deux domaines. Les travaux de Habermas relatifs à l'éthique de la discussion permettent de parer à cette difficulté. Ils montrent que les conditions d'acceptabilité des énoncés descriptifs et normatifs varient selon les types de discours, théoriques et pratiques, où ils sont thématisés, qui ont chacun leurs visées propres. Mais les règles des discours, spécifiées par les conditions idéales de délibération et les présuppositions pragmatiques de l'argumentation, seraient les mêmes tant pour les délibérations théoriques que pour les délibérations pratiques. Le dualisme entre raison théorique et raison pratique retrouve donc, pourrait-on dire, une unité supérieure au niveau des conditions formelles ou procédurales de l'argumentation. Les philosophes descriptivistes cherchent pour leur part à surmonter le dualisme entre raison théorique et raison pratique en imposant les mêmes conditions de vérité à tous les énoncés, tout en refusant aux expressions de forme non descriptive, comme les normes, un lien avec la vérité. Ils privent ainsi ces expressions, qui forment une composante centrale du langage moral, d'une connexion possible avec d'autres standards de validité leur permettant de conserver une valeur épistémique. L'avantage de l'approche défendue par Habermas est de tenter de surmonter le dualisme entre raison théorique et raison pratique, non par l'identité des conditions de vérité, mais par l'identité des conditions d'acceptabilité des énoncés, c'està-dire par l'identité des procédures intersubjectives de délibération permettant aux locuteurs et aux auditeurs de prendre en considération, non plus un

3. On sait que Habermas ajoute aux mondes objectif et social le monde subjectif des états psychologiques internes. Voir à ce sujet la comparaison qu'il fait de sa conception avec la doctrine des trois mondes de Karl Popper (Habermas 1984: 76-84). Charles Larmore (1996) développe une stratégie similaire pour contrer les tendances réductionnistes du naturalisme en différenciant les faits naturels, psychologiques et normatifs. 
seul, mais différents aspects de la validité et de la rationalité (la vérité des propositions et la justesse des normes).

Il est fort possible que Finlayson rejettera les solutions de Habermas aux deux difficultés que j'ai mentionnées. Mais il doit concéder que ces difficultés sont réelles, qu'elles touchent au cœur de l'approche descriptiviste des phénomènes moraux qui sous-tend son « cognitivisme métaéthique » et sa critique de l'éthique de la discussion de Habermas. Comme je l'ai mentionné cependant, il n'est pas du tout clair à mes yeux que le "cognitivisme métaéthique » de Finlayson doive nécessairement impliquer le descriptivisme moral. L'idée selon laquelle les jugements moraux sont susceptibles de vérité et expriment des croyances qui aspirent à la vérité est parfaitement acceptable pour les défenseurs de l'éthique de la discussion et de l'approche déontologique de la morale. Ce qui ne l'est pas, c'est l'idée réductrice selon laquelle les normes, et les expressions morales en général, doivent être traitées exactement comme des propositions descriptives et être évaluées de la même manière. En refusant la thèse analogique de Habermas entre la vérité propositionnelle et la justesse normative, et en la soumettant au test que représente FG, Finlayson admet en somme implicitement qu'il considère que le cognitivisme moral n'est possible que sous la forme du descriptivisme moral, et c'est finalement de cette manière qu'il nous faut comprendre son "cognitivisme métaéthique ". Si cela est vrai, alors la thèse de Finlayson selon laquelle la théorie discursive de la morale de Habermas rejette le "cognitivisme métaéthique » s'avère tout à fait fondée. Mais c'est une heureuse chose, non une lacune, puisque, comme j'ai tenté de le montrer, une telle conception se butte à des difficultés peut-être plus importantes que celles que Finlayson dénonce.

\section{III}

Après avoir examiné les principales difficultés posées par le descriptivisme moral eu égard à la stratégie réductionniste qu'elle épouse lorsqu'il est question des jugements normatifs, j'aimerais maintenant examiner le véritable défi auquel, à mon sens, l'éthique de la discussion est susceptible d'être confrontée lorsqu'il est question de la différenciation entre la vérité et la justesse. Deux objections principales pourraient être soulevées par les défenseurs du descriptivisme moral contre une telle distinction. J'analyse chacune d'elles tour à tour et je leur apporte une réplique provisoire.

Tout d'abord, les défenseurs du descriptivisme moral pourraient attirer l'attention sur certaines asymétries troublantes entre la vérité et la justesse, montrant que cette dernière a toutes les caractéristiques d'un prédicat ordinaire, alors que seule la première semble posséder une véritable dimension épistémique. On ne peut dire "Il est vrai de faire $x$ » ou « $X$ est vrai» mais seulement «Il est vrai que $p$ » ou « $p$ est vrai ». Par contraste, on peut parfaitement dire "Il est juste de faire $X$ » ou « $x$ est juste». Et cela montre que « juste » est un prédicat ordinaire attribué à certaines sortes de sujets référant 
aux choses et aux événements se produisant dans le monde, alors que seule la "vérité » est un prédicat épistémique attribuable aux propositions.

La façon la plus directe de répondre à cette objection est naturellement de ré-établir la symétrie formelle entre les deux types d'énoncés. Il est possible de dire "Il est juste de faire $x$ », mais aussi «Il est juste que $p$ ", et ainsi nous nous retrouvons devant la même structure d'énoncé qu'avec le prédicat de vérité. Mais est-ce que cette stratégie ré-établit automatiquement la dimension épistémique du prédicat de justesse ? L'analyse la plus simple montre le contraire. Nous pouvons dire «Il est vrai que $p$ » ou «Il est juste que $p$ », mais aussi «Il est merveilleux, compréhensible, sage, discutable... que $p$ ». Comme cet exemple le démontre, à toute proposition $p$ nous pouvons attacher, sinon n'importe laquelle, à tout le moins un grand nombre des propriétés que l'on attribue aux choses et aux événements. Cependant, si l'on peut attribuer à peu près tout ce que l'on veut aux propositions, nous ne pouvons attribuer le prédicat de vérité à tout ce que l'on veut : seules les propositions peuvent être dites vraies ou fausses (et les croyances et les jugements qu'elles expriment), non les choses et les événements. L'asymétrie entre vérité et justesse n'est donc en aucun cas surmontée. Car contrairement au "vrai », le « juste» peut être attribué, non seulement aux propositions, mais également à différentes sortes de sujets (aux personnes, aux actions, aux institutions, etc.), comme le fait n'importe quel autre prédicat. Pourquoi alors persister à considérer le « juste » comme un prédicat du second degré analogue à la vérité et ayant les mêmes propriétés épistémiques?

En vue de résoudre ce problème, posons-nous la question suivante: lorsque je dis que quelque chose est juste, par exemple, qu'il est juste que Paul rembourse ses emprunts, qu'est-ce que je veux dire exactement par là ? Qu'est-ce que je considère, exactement, comme juste? Fort probablement, l'action de Paul. Je considère que l'action de rembourser ses dettes est juste, dans le sens de moralement bonne ou recommandable. Si c'est bien ce que je veux signifier par " juste » lorsque j'évalue le comportement de Paul, il convient de se poser cette autre question : qu'est-ce qui fait de l'action de Paul une action moralement bonne ou recommandable ? Qu'est-ce qui la rend telle ? Une simple réflexion révèle que ce ne peut être rien d'autre que la norme sous-jacente à laquelle l'action de Paul emprunte sa valeur morale. Dans le cas présent, une telle norme pourrait être formulée au moyen d'une proposition normative: «On doit rembourser ce que l'on emprunte.» Comme on le voit, la justesse morale et la validité normative de manière générale ne sont au bout du compte une propriété de rien d'autre que des normes, qui nous disent comment le monde doit être, alors que la vérité est une propriété des énoncés déclaratifs qui nous disent comment le monde est. Il est vrai qu'une asymétrie persiste entre le vrai et le juste : la vérité n'est pas, et ne peut être, un attribut des choses et des événements, mais uniquement celui des propositions faites à leur sujet, alors que le juste peut être attribué aux personnes, aux actions, aux institutions, etc. Toutefois, le «normativement juste» est plus qu'un prédicat ordinaire en ce qu'il ne peut être attribué à tout ce que l'on veut : 
il est en premier lieu un attribut des normes, qui forment une réalité idéale, et ce n'est que de manière dérivée que ce prédicat devient un attribut des personnes, de leurs actions, des institutions, etc. La justesse normative ne constitue donc pas un prédicat ordinaire et ne se comporte pas de la même manière que d'autres prédicats comme "jaune ", "beau ", " plaisant ", etc. Alors que ces derniers renvoient, tout au moins selon les philosophes intuitionnistes, à certaines propriétés dispositionnelles des objets (auxquelles nous avons accès lorsque nous avons la sensibilité appropriée), la justesse normative est une propriété des normes et de rien d'autre (et ne nous devient accessible que par la réflexion et la délibération morale). Les objets dans le monde que l'on considère justes, moralement corrects ou recommandables ne font seulement qu'emprunter cette propriété à la validité normative ou déontologique des normes. Et c'est pourquoi, en dépit de l'asymétrie existant entre la vérité propositionnelle et la justesse normative, il fait toujours sens de considérer cette dernière comme ayant une dimension épistémique non repérable dans les prédicats ordinaires que nous utilisons pour décrire le monde ${ }^{4}$.

Pour résumer l'essentiel de ma réplique à la première objection : si les avocats du descriptivisme moral ont raison de souligner l'asymétrie qui existe entre la vérité propositionnelle et la justesse normative, ils ont néanmoins tort de considérer cette dernière comme un simple prédicat ordinaire privé de toute valeur épistémique. Je passe maintenant à l'autre objection que les descriptivistes ne manqueront sûrement pas d'adresser à la thèse analogique de Habermas.

Toujours en vue de faire ressortir la dimension épistémique unique et incomparable de la vérité comparée à d'autres standards de validité, les philosophes descriptivistes s'opposeront à la thèse de l'analogie en faisant valoir l'argumentation suivante: il est possible de dire «Il est vrai qu'il est juste de faire $x$ » sans redondance apparente. $S i$ « juste » possédait réellement une valeur épistémique analogue à la vérité, l'énoncé précédent serait d'une manière ou d'une autre redondant. Puisque ce n'est pas le cas, «juste» doit être entendu en un autre sens que le sens épistémique que l’on accorde normalement à la vérité.

Pour répondre à cette objection, remplaçons pour les fins de l'argument l'énoncé précédent par un autre où «juste» est attribué, non à un verbe

4. À ma connaissance, Habermas a développé une argumentation similaire à la mienne à une seule occasion (Habermas, 1990: 86-94). Étant donné la centralité de la thèse de l'analogie dans son œuvre, et l'importance de l'asymétrie qui subsiste entre vérité et justesse, il est surprenant que cette ligne d'argumentation n'ait pas été approfondie ailleurs. La seule asymétrie sur laquelle s'est penché Habermas récemment touche aux relations entre la justesse normative et la notion d'acceptabilité idéalement justifiée. Alors que cette notion ne serait pas suffisante pour épuiser le sens que nous attribuons normalement à la vérité des propositions, elle serait constitutive de la justesse normative (Habermas 2003). Je ne discuterai pas ici de l'asymétrie entre vérité et justesse sous cet angle. Pour une analyse approfondie de ses forces et faiblesses, voir Lafont (2004). 


\section{$576 \cdot$ Philosophiques / Automne 2008}

d'action, mais à une proposition normative (puisque, comme nous l'avons expliqué précédemment, les actions humaines dérivent leur valeur morale d'une norme sous-jacente). Nous aurons ainsi : «Il est vrai qu'il est juste que... $\mathrm{O} p$ » $\left(\mathrm{O} p\right.$ signifiant «Il est requis, ou obligatoire, que $\left.p »^{5}\right)$. N'y a-t-il réellement aucune redondance ? Cela dépend. En effet, l'énoncé «Il est vrai qu'il est juste que $\mathrm{O} p$ » peut avoir deux sens :

1. La norme Op existe ou prévaut dans une société donnée, ou dans de nombreuses institutions humaines. C'est un fait naturel (social).

2. La norme Op est juste, moralement recommandable. Elle exprime un fait normatif.

Si nous voulons dire (1), alors l'énoncé «Il est vrai qu'il est juste que $\mathrm{O} p$ » est ambigu. Si statuer à propos d'un fait social est réellement ce que nous voulons faire, alors il convient de supprimer " il est juste » en vue d'exprimer clairement notre proposition, laquelle sera autrement confuse. Si par contre nous voulons dire (2) et cherchons à établir un fait normatif, dire alors «Il est vrai qu'il est juste que. .. » crée une redondance inutile. Dire « Il est juste que... » suffit, puisque le prédicat de justesse a précisément pour rôle de remplacer le prédicat de vérité, ou plus précisément, de spécifier en quel sens nous voulons dire que la norme a une valeur de vérité. Finalement, ou bien l'énoncé «Il est vrai qu'il est juste que $\mathrm{O} p$ » ne contient pas de redondance, mais son sens demeure confus en ce que nous ne savons pas si nous cherchons à établir un fait naturel ou un fait normatif; ou son sens est clair, mais seulement dans la mesure où il est redondant, c'est-à-dire que le prédicat «juste » est utilisé en lieu et place du prédicat de vérité en vue d'établir la valeur déontologique de la norme, et de spécifier en quel sens nous la considérons comme vraie ou valable.

Comme le montre l'exemple précédent, la démonstration de redondance peut au mieux être faite si nous évitons les énoncés qui contiennent des verbes d'action, comme dans «Il est vrai qu'il est juste de faire $x$ » où, le prédicat «vrai » ne pouvant être attribué à un verbe d'action, les possibilités de confusion avec «juste» semblent inexistantes. Dans de tels énoncés, l'absence (apparente) de redondance vient du fait que "juste» est utilisé comme un prédicat ordinaire analogue à " rassurant », " plaisant », etc., comme dans des énoncés tels que "Il est vrai qu'il est rassurant, plaisant... de faire $x$ ». Par contraste, le prédicat de justesse utilisé dans notre exemple précédent l'est, non plus simplement en tant que prédicat ordinaire, mais en tant que prédicat du second degré, analogue à la vérité, et attribué primitivement aux normes et seulement de manière dérivée aux actions humaines. Les philosophes descriptivistes objecteront sans doute que des prédicats tels que « rassurant» ou

5. J'utilise le symbolisme standard utilisé en logique déontique. « $\mathrm{O}$ » symbolise une obligation et fonctionne comme un opérateur déontique appliqué à une proposition. Pour une explication détaillée de ce symbolisme, voir von Wright (1983: 100-107). 
"plaisant» peuvent également être attribués aux propositions normatives comme dans "Il est vrai qu'il est rassurant, plaisant... que $\mathrm{O} p$ ». Mais un tel énoncé contraste avec un énoncé similaire contenant le prédicat de justesse de deux manières : (1) Il n'est clairement pas redondant et aucune possibilité de confusion avec le prédicat de vérité ne peut survenir. En d'autres termes, il est clair dès le départ qu'un tel énoncé cherche à établir un fait naturel (social ou psychologique), alors que l'utilisation du prédicat de justesse révèle une ambiguité quant au type de fait (naturel ou normatif) que l'on cherche à établir ; (2) Des prédicats tels que " rassurant ", " plaisant ", etc., ne peuvent d'aucune manière remplacer "vrai » en vue d'exprimer plus précisément le sens selon lequel la norme nous apparaît avoir une valeur de vérité, comme nous pouvons le faire avec le prédicat « juste».

Finalement, le problème avec la seconde objection des descriptivistes est qu'elle attire l'attention uniquement sur les énoncés où «juste » fonctionne comme un prédicat ordinaire attribué à des actions ou à des institutions et ignore son utilisation en relation avec les propositions normatives où les possibilités d'analogie avec la vérité deviennent possible.

\section{Conclusion}

J'aimerais résumer les principaux résultats auxquels je suis parvenu dans cet article. Tout d'abord, il a été démontré que le soi-disant problème de FregeGeach est de peu de pertinence pour rendre compte adéquatement, non seulement des difficultés spécifiques de la thèse analogique entre la vérité et la justesse avancée par la conception discursive de la morale de Habermas, mais également des mérites et démérites relatifs de la conception pragmatique de la vérité qui lui sert d'assise. En second lieu, il a été démontré que la théorie morale de Habermas n'est d'aucune façon opposée à l'idée que les jugements moraux sont susceptibles de vérité et expriment des croyances aspirant à la vérité, mais seulement à l'idée plus limitée que les jugements moraux sont susceptibles de vérité dans le même sens que le sont les jugements descriptifs. La théorie morale de Habermas est uniquement opposée à l'interprétation descriptiviste du cognitivisme moral. Si la thèse centrale de Finlayson est que le cognitivisme moral n'est possible que sous la forme du descriptivisme moral, et si c'est ce que l'on doit entendre par le "cognitivisme métaéthique » auquel il souscrit, alors Habermas a raison de s'y opposer pour la simple raison que cette position ne fait que remplacer les difficultés de son non descriptivisme par d'autres difficultés aussi, sinon plus sérieuses. Finalement, il a été démontré qu'il est possible de contourner certaines objections clés portées à la thèse de l'analogie entre vérité et justesse de telle manière que ses intuitions essentielles soient préservées.

J'aimerais en terminant exprimer une dernière remarque. Le principal point de focalisation de cet article a été les relations entre le cognitivisme moral et le descriptivisme. Il a été démontré qu'un cognitivisme moral non-descriptiviste est possible, que la théorie discursive de la morale de Habermas en forme sans doute le meilleur exemple actuel et que cette dernière peut résister à la 
plupart des objections soulevées contre elle en provenance des philosophes descriptivistes, au nombre desquels figurent des chercheurs comme Finlayson. Mais rien n'a été dit des relations entre le cognitivisme moral et le réalisme moral. Un cognitivisme moral non réaliste est-il viable de manière générale ? En particulier, le type de cognitivisme moral non réaliste développé par Habermas sous la forme du constructivisme moral constitue-t-il une doctrine viable ? Le non-réalisme moral n'affaiblit-il pas considérablement la thèse de l'analogie et ne creuse-t-il pas encore davantage l'écart existant entre vérité et justesse de manière à rendre l'idée même d'une analogie entre elles inutile ? Certains chercheurs le pensent (Lafont 2004). Les objectifs de mon texte étant plus limités, je dois laisser ces questions importantes de côté. Il demeure cependant qu'une démonstration vraiment complète et satisfaisante du bien-fondé de la thèse analogique aurait à prendre en compte, non seulement les relations entre le cognitivisme moral et le descriptivisme, mais également celles entre le cognitivisme moral et le réalisme moral.

\section{Références}

Apel, K.-O. (1998a) «The a priori of the Communication Community and the Foundations of Ethics: The Problem of a Rational Foundation of Ethics in the Scientific Age ", dans K.-O. Apel, Towards a Transformation of Philosophy (Milwaukee, Wisconsin, Marquette University Press).

. (1998b) «Transcendental Semiotics and the Paradigms of First Philosophy ", dans M. Papastephanou (éd.), From a Transcendental-Semiotic Point of View, p. 43-63 (Manchester, Manchester University Press).

. (1998c) "Can an Ultimate Foundation of Knowledge Be Non-Metaphysical ? ", dans M. Papastephanou (éd.), From a Transcendental-Semiotic Point of View, p. 81-102 (Manchester, Manchester University Press).

ـ (1989) «Normative Begründung der 'Kritischen Theorie' durch Rekurs auf lebensweltliche Sittlichkeit ? Ein transzendental-pragmatisch orientierter Versuch, mit Habermas gegen Habermas zu denken ", dans A. Honneth, T. McCarthy, C. Offe \& A. Wellmer (éd.), Zwischenbetrachtungen. Im Prozess der Aufklärung, p. 15-65 (Frankfurt, Suhrkamp).

_ (1987) «Fallibilismus, Konsenstheorie der Wahrheit und Letztbegründung », dans Forum für Philosophie Bad Homburg (éd.), Philosophie und Begründung, p. 116-211 (Frankfurt, Suhrkamp).

Ayer, A. (1936) Language, Truth and Logic (New York, Dover Publications). Blackburn, S. (1984) Spreading the Word (Oxford, Oxford University Press).

Dancy, J. (2004) Ethics Without Principles (Oxford, Clarendon Press).

Finlayson, J. G. (2005) «Habermas's Moral Cognitivism and the Frege-Geach Challenge ", European Journal of Philosophy, 13, p. 319-344.

Frege, G. (1970) «Negation ", dans P. Geach \& M. Black (éd.), Translations from the Philosophical Writings of Gottlob Frege, p. 117-137 (Oxford, Basil Blackwell). Geach, P. T. (1965) «Assertion », Philosophical Review, 74, p. 449-445.

Habermas, J. (2003) «Rightness versus Truth: On the Sense of Normative Validity in Moral Judgements and Norms ", dans J. Habermas, Truth and Justification, p. 237-276 (Cambridge, Polity Press). 
. (1998) "What is Universal Pragmatics?", dans M. Cooke (éd.), On the Pragmatics of Communication, p. 21-103 (Cambridge, Mass., The MIT Press). . (1996) Between Facts and Norms. Contributions to a Discourse Theory of Law and Democracy (Cambridge, Mass., The MIT Press).

- (1993) «Remarks on Discourse Ethics ", dans J. Habermas, Justification and Application. Remarks on Discourse Ethics (Cambridge, Mass., The MIT Press). - (1990) "Discourse Ethics. Notes on a Program of Philosophical Justification ", dans J. Habermas, Moral Consciousness and Communicative Action, p. 43-115 (Cambridge, Mass., The MIT Press).

. (1986) «Entgegnung », dans A. Honneth \& H. Jonas (éd.), Kommunikatives Handeln. Beiträge zu Jürgen Habermas' "Theorie des kommunikativen Handelns ", p. 327-405 (Frankfurt, Suhrkamp).

. (1984) The Theory of Communicative Action (Boston, Beacon Press).

Lafont, C. (2004) «Moral Objectivity and Reasonable Agreement: Can Realism Be Reconciled with Kantian Constructivism? ", Ratio Juris, 17, p. 27-51.

Larmore, C. (1996) «Moral Knowledge », dans C. Larmore, The Morals of Modernity, p. 89-117 (Cambridge, Cambridge University Press).

Stevenson, C. (1944) Ethics and Language (Yale, Yale University Press).

von Wright, G. H. (1983) Practical Reason (Ithaca, New York, Cornell University Press. 